\title{
ARTICLE OPEN \\ Path to stable quantum spin liquids in spin-orbit coupled correlated materials
}

\author{
Andrei Catuneanu ${ }^{1}$, Youhei Yamaji ${ }^{2,3}$, Gideon Wachtel ${ }^{1}$, Yong Baek Kim ${ }^{1,4}$ and Hae-Young Kee ${ }^{1,4}$
}

The spin liquid phase is one of the prominent strongly interacting topological phases of matter whose unambiguous confirmation is yet to be reached despite intensive experimental efforts on numerous candidate materials. Recently, a new family of correlated honeycomb materials, in which strong spin-orbit coupling allows for various bond-dependent spin interactions, have been promising candidates to realize the Kitaev spin liquid. Here we study a model with bond-dependent spin interactions and show numerical evidence for the existence of an extended quantum spin liquid region, which is possibly connected to the Kitaev spin liquid state. These results are used to provide an explanation of the scattering continuum seen in neutron scattering on $a$ - $\mathrm{RuCl}_{3}$.

npj Quantum Materials (2018)3:23; doi:10.1038/s41535-018-0095-2

\section{INTRODUCTION}

The role of strong interaction between electrons in the emergence of topological phases of matter is currently a topic of intensive research. The archetypal example of a topological phase with strong electron interaction is the quantum spin liquid, ${ }^{1}$ in which the elementary excitations are charge-neutral fractionalized particles. While a lot of progress has been made on the theoretical understanding of the quantum spin liquid phase, its direct experimental confirmation has remained elusive despite various studies on a number of candidate materials. ${ }^{2-6}$ Significant progress, however, has recently been made due to the availabilty of a new class of correlated materials, where strong spin-orbit coupling leads to various bond-dependent spin interactions, ${ }^{7-9}$ thus resulting in magnetic frustation. These materials are Mott insulators with $4 d$ and $5 d$ transition metal elements, which include iridates and ruthenates ${ }^{10-14}$ and come in two-dimensional (2D) or three-dimensional (3D) honeycomb variants. They have been particularly exciting because they intrinsically have a strong Kitaev interaction and therefore could potentially realize the Kitaev spin liquid (KSL) phase-an example of a $\mathbb{Z}_{2}$ quantum spin liquid where the electron's spin $-\frac{1}{2}$ fractionalizes into two degrees of freedom: itinerant Majorana fermions and $\mathbb{Z}_{2}$ fluxes.

While the Kitaev interaction $(K)$ in these materials is large, it competes with symmetry allowed nearest-neighbor (n.n.) symmetric off-diagonal $(\Gamma)$ and Heisenberg $(J)$ spin interactions. ${ }^{15}$ For example, in $a-\mathrm{RuCl}_{3}\left(\mathrm{RuCl}_{3}\right)$, an actively studied $\mathrm{KSL}$ candidate, comprehensive $a b$ initio computations and recent dynamical studies $^{16,17}$ suggest that ferromagnetic $K$ and antiferromagnetic $\Gamma$ interactions are dominant and comparable in magnitude, while $J$ is negligible. ${ }^{18-20}$ The balance of these and additional small further neighbor interactions causes $\mathrm{RuCl}_{3}$ and other $\mathrm{KSL}$ candidates to magnetically order at low temperature; however, it is still unclear whether or not the often-large $\Gamma$ interaction prefers magnetic ordering. Meanwhile, the community has attempted to revive the possibility of a $\mathrm{KSL}$ in $\mathrm{RuCl}_{3}$ by applying a small magnetic field, with the effect of entering a potential spin liquid phase with no magnetization. ${ }^{20}$

Since a weak magnetic field takes $\mathrm{RuCl}_{3}$ out of the ordered phase, it lends credence to the idea that the zig-zag phase is stabilized by small interactions at comparable energy scale to the magnetic field, such as a $3 r d$ n.n. Heisenberg $J_{3}{ }^{18-20}$ term or terms coming from slight trigonal distortion. ${ }^{21}$ This calls into question the role of the $\Gamma$ interaction. Interestingly, a recent analysis of the $\Gamma$ model revealed a macroscopically degenerate classical ground state. $^{22}$

In this work we will thus investigate if a model with $K$ and $\Gamma$ hosts an extended quantum spin liquid phase. A previous exact diagonalization (ED) study on a 24-site honeycomb cluster hints that the ferromagnetic KSL is unstable after perturbing with a small $\Gamma$, but the resulting phase is not orderered. ${ }^{15}$ On the other hand, it is known that the KSL is stable upon introducing bond anisotropy, which is present in real materials as depicted in Fig. 1 a. We indeed find that such anisotropy can extend the KSL phase between the $-K$ and $\Gamma$ limits, as shown in Fig. $1 \mathrm{~b}$.

We consider the following nearest-neighbor (n.n.) model on a 2D honeycomb lattice:

$H=\sum_{\gamma \in X, y, z} H^{\gamma}$,

where

$H^{z}=\sum_{\langle i j\rangle \in z-\text { bond }}\left[K_{z} S_{i}^{z} S_{j}^{z}+\Gamma_{z}\left(S_{i}^{x} S_{j}^{y}+S_{i}^{y} S_{j}^{x}\right)\right]$

and $H^{x, y}$ are defined similarly with corresponding $K_{x, y}$ and $\Gamma_{x, y}$. Each $H^{\gamma}$ represents the n.n spin interactions along one of the three bond directions, $\gamma=x, y, z$. The model is parameterized by $K_{z}=-\left(1+2 a_{K}\right) \cos \phi, K_{x, y}=-\left(1-a_{K}\right) \cos \phi, \Gamma_{x, y, z}=\sin \phi$, with $a_{K}$ characterizing bond anisotropy. When $\phi=0, \pi$ (i.e., $\Gamma_{\gamma}=0$ ), this model reduces to the exactly solvable Kitaev model with the KSL ground state.

\footnotetext{
'Department of Physics and Center for Quantum Materials, University of Toronto, 60 St. George St., Toronto, ON M5S 1A7, Canada; ${ }^{2}$ Department of Applied Physics and QuantumPhase Electronics Center (QPEC), The University of Tokyo, Hongo, Bunkyo-ku, Tokyo 113-8656, Japan; ${ }^{3}$ JST, PRESTO, Hongo, Bunkyo-ku, Tokyo $113-8656$, Japan and ${ }^{4}$ Canadian Institute for Advanced Research, Toronto, ON M5G 1Z8, Canada

Correspondence: Hae-Young Kee (hykee@physics.utoronto.ca)
}

Received: 17 November 2017 Revised: 12 March 2018 Accepted: 20 March 2018

Published online: 24 April 2018 

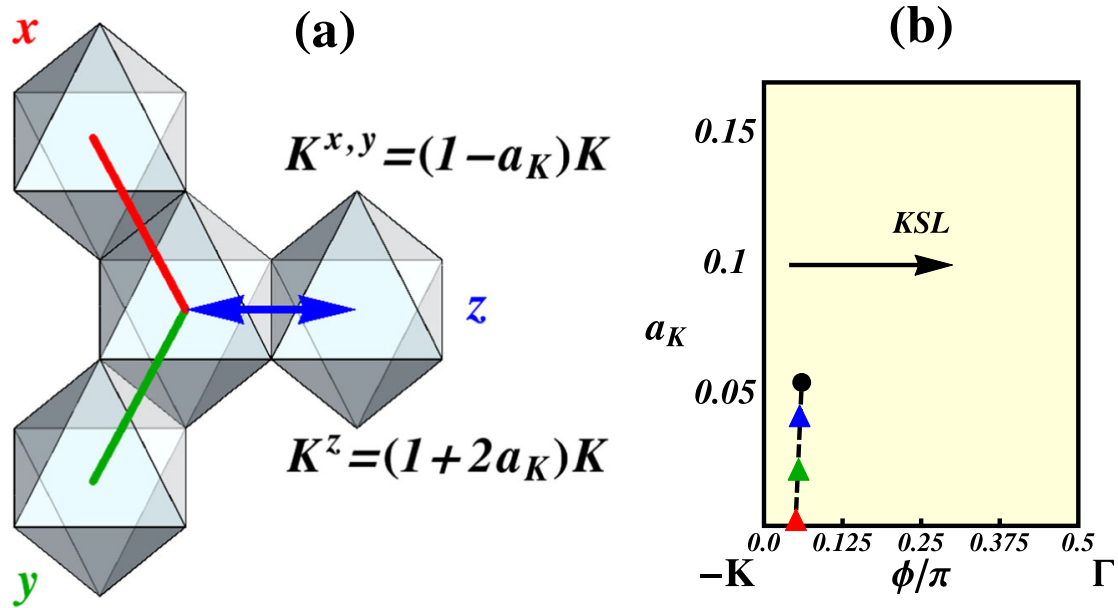

Fig. 1 a Shorter $z$-bond leading to stronger $K^{z}$ and weaker $K^{x, y}$ interactions, parameterized by $a_{K}$ in Eq. 1. b $a_{K}$ phase diagram of Eq. 1. A line of first order phase transitions (dashed black line), terminating below $a_{K}=0.06$ (black dot), separates the $-K$ and $\Gamma$ limits. Anisotropy allows the KSL to be adiabatically connected to the $\Gamma$-phase (arrow). Red, green, and blue triangles indicate first-order phase transitions when $a_{K}=0.0,0.02$, and 0.04 respectively, as seen in $N=18$ and 24-site exact diagonalization calculations. A subsequent iDMRG calculation ${ }^{38}$ has further corroborated the phase transition labeled by the red triangle

(a)

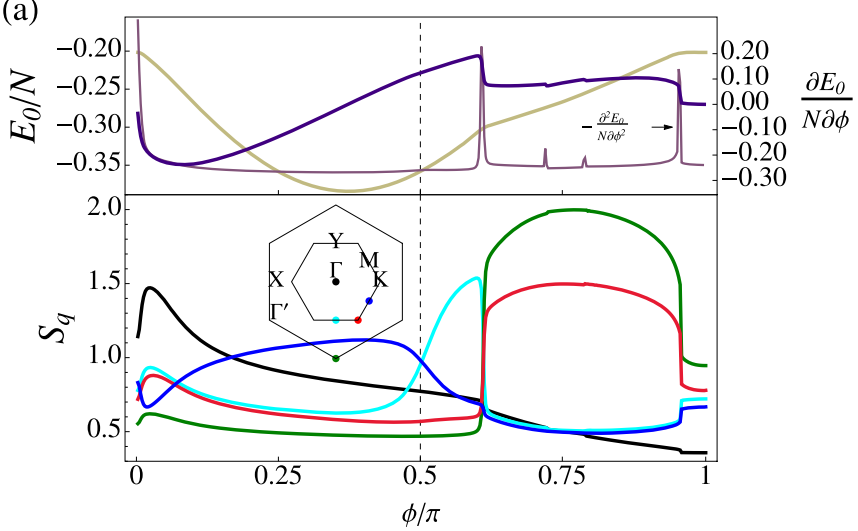

(b)

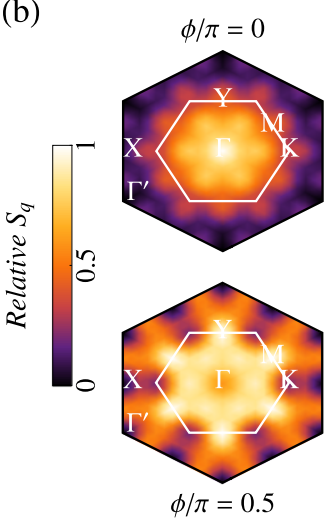

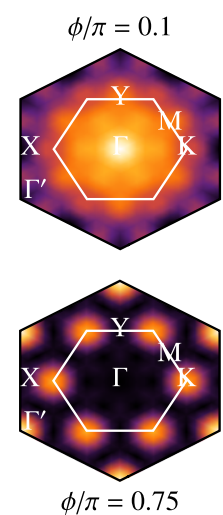

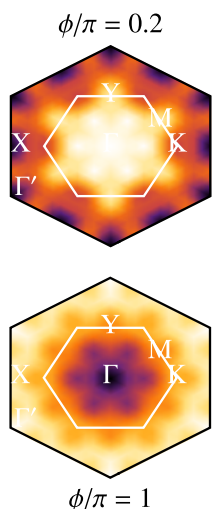

Fig. 2 a Top: $E_{0} / N$ (yellow), $\frac{1}{N} \frac{\partial E_{0}}{\partial \phi}$ (purple), and $-\frac{1}{N} \frac{\partial^{2} E_{0}}{\partial \phi^{2}}$ (light purple) for anisotropy parameter $a_{K}=0.1$. Bottom: $S_{q}$ for anisotropy parameter $a_{K}=0.1$ at $\Gamma$ (black), M (blue), $Y$ (cyan), $\mathrm{K}$ (red), and $\Gamma$ (green) in the reciprocal lattice (inset). b Representation of $S_{q}$, averaged over domains in a real material, when $a_{K}=0.1$ for various $\phi$ in the phase diagram

We have studied this model using a combination of three different, corroborating, numerical methods: ED on a 24-site honeycomb cluster, the method of thermal pure quantum states, ${ }^{23-27}$ and infinite time-evolution block decimation (iTEBD). We have first reproduced the earlier work in the isotropic $a_{K}=0$ limit, showing a strong first-order transition between $-K$ and $\Gamma$ limits (see Supplementary Materials (SM)). We present the following results using our numerical techniques:

1. When $a_{K} \geq 0.06$, we find that the $-K_{Y}(0 \leq \phi \leq \pi / 2)$ and $\Gamma_{\gamma}(\phi /$ $\pi=0.5$ ) limits are adiabatically connected as shown in Fig. $1 \mathrm{~b}$. Thus we find evidence for an extended quantum spin liquid phase in the presence of anisotropy, $a_{K}$.

2. An intervening magnetically ordered phase separates the spin liquid phase near the pure $\Gamma_{\gamma}$ limit and the antiferromagnetic $\mathrm{KSL}$ at $\phi / \pi=1$.

3. The specific heat $C(T)$ and entropy $S(T)$ at finite temperatures suggest a smooth crossover from the ferromagnetic Kitaev limit to the pure $\Gamma_{\gamma}$ limit, consistent with our ED results.

4. Zig-zag spin correlations become dominant upon perturbing the quantum spin liquid phase in $0<\phi<\pi / 2$ by $J_{3}$, indicating the enhancement of zig-zag order by $J_{3}$.

\section{RESULTS}

Extended spin liquid state in global phase diagram

The ground state energy per site $E_{0} / N$ of Eq. 1 was computed for $\phi / \pi \in(0,1)$, and for different anisotropy parameters by ED on a 24site cluster using periodic boundary conditions (see SM). Discontinuities in $\frac{1}{N} \frac{\partial E_{0}}{\partial \phi}$ were used to identify possible phase transitions. Remarkably, when $\phi / \pi \in(0,0.5)$ and $0 \leq a_{K}<0.06$, there is a line of first order phase transitions that terminate at $a_{K}$ $=0.06$. Above $a_{K}=0.06$, the first derivative of the energy presents no sharp features suggesting that the ground state of the $\Gamma$-limit $(\phi / \pi=0.5)$ is adiabatically connected to the ferromagnetic KSL ( $\phi)$ $\pi=0)$ as depicted in Fig. $1 \mathrm{~b}$ for $a_{K}=0.1$.

In the antiferromagnetic region of phase space, there are two large discontinuities in $\frac{1}{N} \frac{\partial E_{0}}{\partial \phi}$ that encompass a large region of phase space separating the $\Gamma$-limit and the exactly solvable antiferromagnetic Kitaev limit at $\phi / \pi=1$. These peaks coincide with kinks in $E_{0} / N$ (solid yellow) shown in Fig. 2a. Two smaller discontinuities can also be seen near $\phi / \pi=0.75$, however these are not present when $a_{k}=0$, while the larger jumps near $\phi / \pi=0.5$ and 1 appear consistently for different $a_{k}$. The small discontinuities can thus be considered spurious and a consequence of the finite 


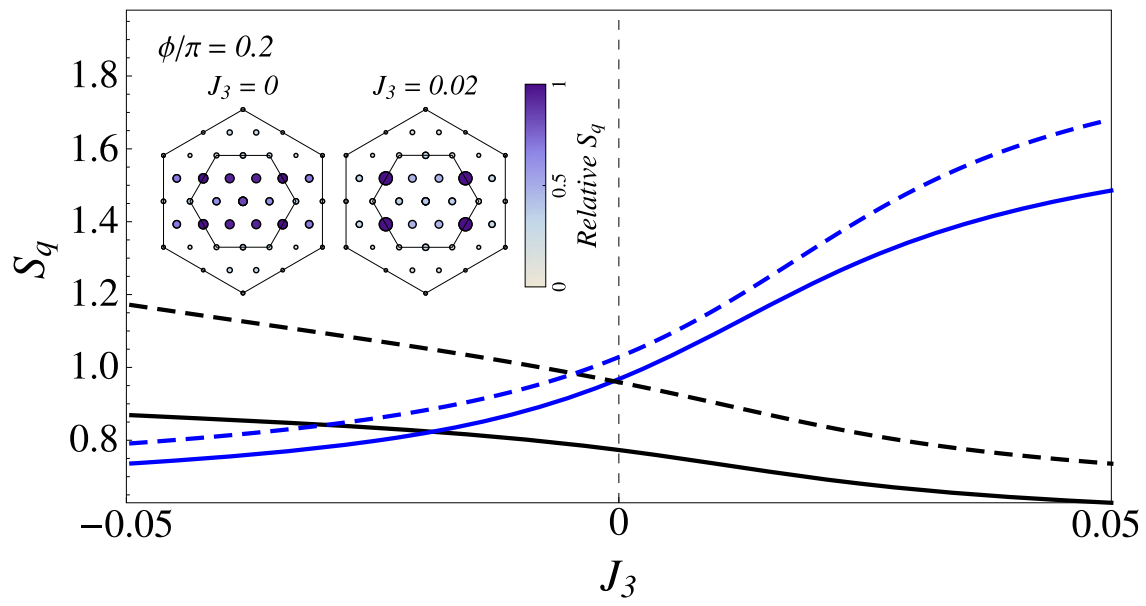

Fig. 3 SSF at the $\Gamma$-points (black) and M-points (blue) in the BZ for small $J_{3}$. Solid and dashed curves correspond to $\phi / \pi=0.5$ and 0.2 , respectively. The inset shows the dramatic change in the relative intensity at the $\Gamma$-points and M-points for small $J_{3}>3$

cluster size. Similar finite size effects were also found for $\phi / \pi \in(0$, $0.5)$ when $a_{K}=0$, as discussed in the SM.

\section{Magnetic order and perturbations}

The ground state wavefunction of Eq. 1 computed by ED is used to evaluate real-space spin-spin correlation functions $\left\langle S_{i} \cdot S_{j}\right\rangle$, where $i$ and $j$ are site indices on the honeycomb lattice. By Fourier transform, we obtain the static structure factor (SSF) given by $S_{q}=\frac{1}{N} \sum_{i, j} e^{i\left(r_{i}-r_{j}\right) \cdot q}\left\langle S_{i} \cdot S_{j}\right\rangle$, where $q$ is a vector in the reciprocal lattice. The SSF at various points in the Brillouin zone (BZ) is plotted over the phase space in the bottom panel of Fig. 2a.

The discontinuities in the SSF can be directly matched with those in $\frac{1}{N} \frac{\partial E_{0}}{\partial \phi}$. Visualizations of the SSF over the BZ for representative $\phi$ in the phase diagram are presented in Fig. $2 b$. The SSF in Fig. $2 b$ is obtained by computing the average of $\left\langle S_{i} \cdot S_{j}\right\rangle$ over all n.n. bonds, 2nd n.n., etc. This calculation reflects the presence of different domains in the crystal, in which either of $x, y$, $z$ bond interactions can be stronger and thus, over the whole crystal, these domains result in an isotropic $S_{q}$ despite the inherent bond anistropy in Eq. 1. The SSF varies adiabatically when $a_{K}=0.1$ for $\phi \in(0, \pi / 2)$ and the spin correlations at the $\Gamma$-points and $M$ points are comparable in intensity when $\Gamma \simeq K_{\gamma}$, leading to a "star"-shaped structure in the SSF as seen in Fig. 2 b (e.g., $\phi / \pi=$ 0.2 ). The extended phase separating $\phi / \pi=0.5$ and 1 is characterized by dominating spin correlations at the K-points and $\Gamma^{\prime}$-points in the reciprocal lattice $(\phi / \pi=0.75$ in Fig. $2 b)$. Contained within this phase is the exactly solvable point with hidden $S U(2)$ symmetry at $\phi / \pi=0.75$, which features K-point correlations ${ }^{28}$ consistent with the results presented here. Thus the extended spin liquid phase for ferromagnetic $\mathrm{K}$ is separated from the antiferromagnetic KSL at $\phi / \pi=1$ by a magnetically ordered phase.

These results can be connected to real materials, particularly $\mathrm{RuCl}_{3}$ in which a zig-zag magnetic ordering has been observed. ${ }^{29-}$

31 Previous studies have shown that in addition to the n.n. ferromagnetic Kitaev and antiferromagnetic $\Gamma$ interactions, a 3rd $n$. n. antiferromagnetic Heisenberg interaction $J_{3} \sum_{\langle\langle\langle i, j\rangle\rangle\rangle} S_{i} \cdot S_{j}$ is non-vanishing and plays a role in determining the magnetic ordering in $\mathrm{RuCl}_{3}{ }^{18,19}$ Figure 3 shows that the effect of perturbing Eq. 1 by $J_{3}$ is to enhance (suppress) the M-point ( $\Gamma$-point) spin correlations, consistent with a zig-zag magnetically ordered state observed in experiments. This result indicates that by tuning $J_{3}$ in the real material, an alternate path to achieve a spin liquid phase may be realized.

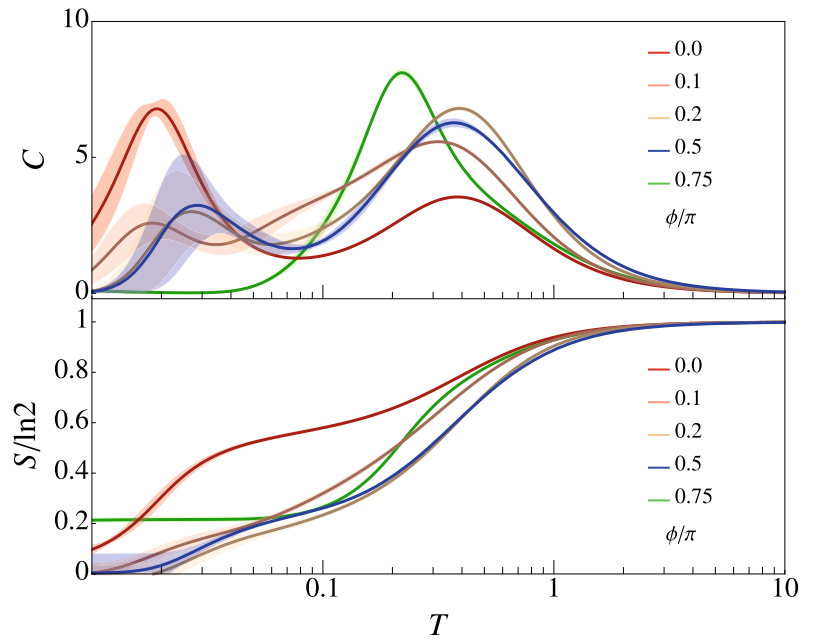

Fig. 4 Results of the method of thermal pure quantum states on a 24-site cluster with anisotropy parameter $a_{K}=0.1$. Plotted in solid curves are the temperature dependence of heat capacity $C(T)$ (top) and entropy $S(T)$ (bottom) for various $\phi$ in the phase diagram. The shaded regions represent the estimated errors on the results. The temperature $\Gamma$ is expressed in units, where $\sqrt{\left(K_{z} /\left(1+2 a_{K}\right)\right)^{2}+\Gamma_{z}^{2}}=$ $\sqrt{\left(K_{x, y} /\left(1-a_{K}\right)\right)^{2}+\Gamma_{x, y}^{2}}=1$

Specific heat and thermal entropy

Previous study on the finite temperature properties of the Kitaev model has shown that KSLs feature two peaks in the heat capacity $C(T)$ and a $\frac{1}{2}-$ plateau in the entropy $S(T)$, which is attributed to the thermal fractionalization of spin degrees of freedom. ${ }^{32}$ Here we go beyond the Kitaev limit and investigate the heat capacity and entropy at finite temperature in the presence of $\Gamma$, which is expected to compete with $K_{y}$ in $\mathrm{RuCl}_{3}$, using the method of thermal pure quantum states (see $\mathrm{SM}$ ).

The dependence of $C(T)$ on $\phi$ when $a_{K}=0.1$ is plotted in the top panel of Fig. 4. The expected two peak structure in $C(T)$ is observed when $\phi / \pi=0$, and is seen to be maintained continuously as $\phi / \pi$ approaches 0.5 so that the $\Gamma$-limit shows a qualitatively similar behavior in $C(T)$ to the KSL. Evidence for a phase transition can be seen when $\phi \gtrsim 0.7$ on account of the abrupt change in $C(T)$, resembling that of the heat capacity in trivially ordered phases. ${ }^{33}$ This finding is consistent with our ED results and the 120 -order at $\phi / \pi=0.75$ seen in refs. ${ }^{15} 28$. The 
dependence of $S(T)$ on $\phi$ is plotted in the bottom panel of Fig. 4 with a clear $\frac{1}{2}-$ plateau observed when $\phi / \pi=0$, consistent with the expected KSL behavior. In addition, a plateau of about 1/5 the total entropy is observed when $\phi / \pi=0.5$. Another plateau is observed in the magnetically ordered phase around $\phi / \pi=0.75$; however, this feature can be attributed to finite-size effects as follows. The $(N+1)$-fold ground state degeneracy at $\phi / \pi=0.75$ due to the hidden $S U(2)$ symmetry $^{28}$ is only slightly broken away from this point, inducing a plateau in $S(T)$ with height given by $\ln (N+1) / N \ln 2 \simeq 0.1935 \sim 1 / 5$ when $N=24$. By contrast, the height of the plateau around $\phi / \pi=0.5$ is independent of $N$. (The height of the plateau in the temperature dependence of entropy is also examined by using a 32 site cluster).

The physical origin of the two peak structure in $C(T)$ and the plateau in $S(T)$ can be traced to the energy scales of the thermal fluctuations of the underlying quasiparticles in the spin liquid. ${ }^{32}$ In the KSL at zero temperature, the low-lying quasiparticle excitations are characterized by itinerant Majorana fermions which disperse in a background of zero flux. ${ }^{34,35}$ It has been shown that as temperature increases, the flux degrees of freedom begin to fluctuate and lead to the low temperature peak seen in $C(T)$, resulting in the plateau seen in $S(T)$. Furthermore, the high temperature peak in $C(T)$ is attributed to the development of short range spin correlations. ${ }^{32}$ Our results show that the two-peak structure in $C(T)$ is qualitatively maintained and further suggests that no phase transition has taken place.

\section{Similarities on the infinite tree}

We further studied Eq. 1 on an infinite Cayley tree with $z=3$ connectivity, using the iTEBD ${ }^{36}$ (see SM). Classically, the ground state in the $\Gamma$-limit on the infinite tree is macroscopically degenerate because a different state with the same energy can be constructed by flipping the sign of one spin component on an infinite string of neighboring spins. The $\Gamma$-limit on the 2D honeycomb and 3D hyper-honeycomb ${ }^{21}$ lattices also feature similar classical degeneracy. The similarity at the classical level of the $\Gamma$-limit on the infinite tree to the 2D and 3D lattices prompts us to study the quantum model on the infinite tree for further insight.

Figure 5 shows results of the eight-site iTEBD calculation with bond dimension $X=10$, and anisotropy $a_{K}=0.1$. In this calculation, we have also introduced an anisotropy to $\Gamma_{\gamma}$ such that $\Gamma_{x}=\Gamma_{y}$

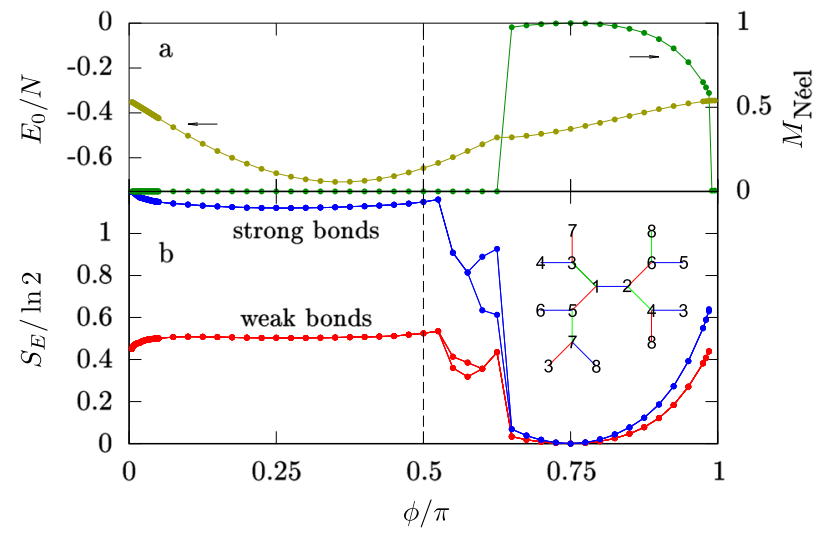

Fig. 5 iTEBD results for small anisotropy $a_{K}=a_{\Gamma}=0.1$, and bond dimension $X=10$. a Néel order parameter $M_{\text {Néel, }}$ and ground state energy per site, $E_{0} / N$. b Entanglement entropy $S_{E}$ associated with splitting the system along the different bonds in the eight-site unit cell. In most of the phase diagram all the strong $z$ bonds are identical, and so are all the weak, $x$ and $y$ bonds. In the transition region near $\phi=0.6 \pi$, the symmetry between like bonds is broken, perhaps indicating a first order transition. At $\phi / \pi=0.75$, the entanglement entropy vanishes since the system is in a product state. Inset: schematic of Cayley tree with $z=3$ connectivity
$=\left(1-a_{\Gamma}\right) \sin \phi$ and $\Gamma_{z}=\left(1+2 a_{\Gamma}\right) \sin \phi$ in order to apply the iTEBD method (see SM). No transition is found when $\phi / \pi \in(0,0.5)$ and the obtained state is a highly entangled paramagnet, with $S_{E} \sim$ 0.8 for strong $(z)$ bonds, while for weak $(x, y)$ bonds, $S_{E} \sim 0.4$. Deep in the gapped phase of the Kitaev model, with large anisotropy $a_{K}$, one finds $S_{E} \sim \log 2 \sim 0.693$ for the strong bonds and much smaller values of $S_{E}$ for the weak bonds. Both, however, increase as the anisotropy is reduced-perhaps due to a finite contributions from the Majorana fermions. ${ }^{37}$ An increase is expected in the entanglement entropy as one approaches a phase transition, however no such peaks are seen for $0<\phi<\pi / 2$. Similarly, there are no sharp features in the ground state energy $E_{0}$ as a function of $\phi$, which indicates that this phase is adiabatically connected to the KSL at $\phi=0$.

There is an apparent first order transition around $\phi=0.6 \pi$ into a Néel state with spins ordered in the (111) direction, accompanied by a dramatic lowering of $S_{E}$ on both strong and weak bonds into this region. This Néel state becomes a simple product state when $\phi / \pi=0.75$, as seen by the vanishing of $S_{E}$. A final transition into a paramagnetic state is seen before the antiferromagnetic Kitaev limit.

\section{DISCUSSION}

The highlight of our numerical results is that, in the presence of bond anisotropy $a_{k}$, there exists an extended quantum spin liquid region which is adiabatically connected to the ferromagnetic KSL. The model we have studied is motivated by experiments on $\mathrm{RuCl}_{3}$ and earlier ab initio computations. ${ }^{18-20}$ In a recent inelastic neutron scattering experiment on $\mathrm{RuCl}_{3}$, it is found that the continuum of finite energy excitations exists both below and above the magnetic transition temperature despite that the low temperature ground state is the zig-zag long-range ordered state. ${ }^{38}$ The inelastic neutron scattering data for the continuum show the star-shape intensity that extends from the zone center towards the M-points of the BZ.

Recall that the SSF in our ED study shows enhanced (decreased) short-range spin correlations at the M-point (zone center) of the $\mathrm{BZ}$ as one moves from the ferromagnetic Kitaev limit to the pure $\Gamma_{\gamma}$ limit. When the strength of the ferromagnetic Kitaev interaction and the $\Gamma_{\gamma}$ interaction become comparable, both of the shortrange spin correlations at the $M$ and the zone center would show significant intensity, which leads to the star-shape structure in momentum space. This behavior may be favorably compared to the finite-energy short-range spin correlations seen in $\mathrm{RuCl}_{3}$. Given that the $a b$ initio computations suggest comparable magnitudes of the ferromagnetic Kitaev and $\Gamma_{\gamma}$ interactions in $\mathrm{RuCl}_{3}{ }^{18}$ it is conceivable that $\mathrm{RuCl}_{3}$ may be very close to the quantum spin liquid phase found in our model and, as shown in our work, the introduction of small $J_{3}$ would favor the zig-zag magnetically ordered phase observed in $\mathrm{RuCl}_{3}$.

Finally, more analytical understanding of the connection between the pure Kitaev limit and the quantum spin liquid phases identified in our numerical work would be extremely valuable for future applications on real materials. Note that a possible incommensurate magnetic ordering cannot be ruled out due to finite cluster size. However, based on the results of a recent iDMRG study, ${ }^{39}$ no evidence of incommensurate ordering allowed by their momentum cuts was found. It is also interesting to note that quantum fluctuations do not lift the infinite ground state degeneracy of the classical model for positive $\Gamma$, while they may lead to incommensurate ordering for negative $\Gamma{ }^{40}$ Thus it is likely that the positive $\Gamma$ regime studied here possesses a spin liquid ground state, and the precise nature of the spin liquid is an excellent topic for future study. 


\section{METHODS}

Our results were obtained using the combination of the three independent numerical techniques listed below.

\section{Exact diagonalization}

Exact diagonalization was performed on a 24-site cluster with periodic boundary conditions. This cluster allows all the symmetries present in the infinite honeycomb lattice and has been used reliably in previous related classical and quantum studies. ${ }^{15,40}$ The Hamiltonian given by Eq. 1 in the main text does not have the $U(1)$ symmetry associated with $S^{z}$ conservation, making it impossible to block diagonalize into magnetization sectors. Therefore, the translational symmetry of the 24-site cluster was used to block diagonalize into different momentum sectors to gain more information about its energy spectrum. The lowest energies and corresponding wavefunctions of each block were then numerically obtained using the Lanczos method. Further details and calculations can be found in the SM.

\section{Thermal pure quantum states}

We used the method of thermal pure quantum states ${ }^{26,27}$ in our specific heat and thermal entropy calculations. A part of the TPQ results were checked by a program package, $H \Phi{ }^{41}$ Details about the construction of thermal pure quantum states and the subsequent calculation of specific heat and entropy can be found in the SM.

\section{Infinite time-evolving block decimation algorithm}

The Hamiltonian given by Eq. 1 was studied on an infinite Cayley tree with $z=3$ connectivity using the iTEBD. ${ }^{36}$ Details about the method and the construction of the ground state can be found in the SM.

Data availability

All relevant data is available from the corresponding author.

\section{ACKNOWLEDGEMENTS}

This work was supported by the NSERC of Canada and the Center for Quantum Materials at the University of Toronto. Y.Y. was supported by JSPS KAKENHI (Grant numbers $15 \mathrm{~K} 17702$ and $16 \mathrm{H} 06345$ ) and was supported by PRESTO, JST. Y.Y. was also supported in part by MEXT as a social and scientific priority issue (creation of new functional devices and high-performance materials to support next-generation industries) to be tackled by using post-K computer. Computations were mainly performed on the GPC supercomputer at the SciNet HPC Consortium. SciNet is funded by: the Canada Foundation for Innovation under the auspices of Compute Canada; the Government of Ontario; Ontario Research Fund-Research Excellence; and the University of Toronto. A part of the TPQ results were checked by a program package, HФ. We thank helpful discussions with Frank Pollmann, Matthias Gohlke, Shunsuke Furukawa, and Subhro Bhattacharjee. We particularly thank Natalia Perkins and loannis Rousochatzakis for informing us of their unpublished ED results on related models.

\section{AUTHOR CONTRIBUTIONS}

A.C. and Y.Y. performed the ED calculations. Y.Y. performed the thermal pure quantum states calculations. G.W. performed the iTEBD calculations. H.Y.K. and Y.B.K. supervised the study. All authors contributed to the writing of the manuscript.

\section{ADDITIONAL INFORMATION}

Supplementary information accompanies the paper on the npj Quantum Materials website (https://doi.org/10.1038/s41535-018-0095-2).

Competing interests: The authors declare no competing interests.

Publisher's note: Springer Nature remains neutral with regard to jurisdictional claims in published maps and institutional affiliations.

\section{REFERENCES}

1. Balents, L. Spin liquids in frustrated magnets. Nature 464, 199-208 (2010).

2. Shimizu, Y., Miyagawa, K., Kanoda, K., Maesato, M. \& Saito, G. Spin liquid state in an organic Mott insulator with a triangular lattice. Phys. Rev. Lett. 91, 107001 (2003).
3. Helton, J. S. et al. Spin dynamics of the spin $-\frac{1}{2}$ Kagome lattice antiferromagnet $\mathrm{ZnCu}_{3}(\mathrm{OH})_{6} \mathrm{Cl}_{2}$. Phys. Rev. Lett. 98, 107204 (2007).

4. Okamoto, Y., Nohara, M., Aruga-Katori, H. \& Takagi, H. Spin-liquid state in the $\mathrm{S}=$ 1/2 hyperkagome antiferromagnet $6 \mathrm{Na}_{4} \mathrm{Ir}_{3} \mathrm{O}_{8}$. Phys. Rev. Lett. 99, 137207 (2007).

5. Yamashita, M. et al. Highly mobile gapless excitations in a two-dimensional candidate quantum spin liquid. Science 328, 1246-1248 (2010).

6. Han, T.-H. et al. Fractionalized excitations in the spin-liquid state of a kagomelattice antiferromagnet. Nature 492, 406-410 (2012).

7. Jackeli, G. \& Khaliullin, G. Mott insulators in the strong spin-orbit coupling limit: from Heisenberg to a quantum compass and Kitaev models. Phys. Rev. Lett. 102, 017205 (2009).

8. Witczak-Krempa, W., Chen, G., Kim, Y. B. \& Balents, L. Correlated quantum phenomena in the strong spin-orbit regime. Annu. Rev. Condens. Matter Phys. 5, 57 (2013).

9. Rau, J. G., Lee, E. K.-H. \& Kee, H.-Y. Spin-orbit physics giving rise to novel phases in correlated systems: iridates and related materials. Annu. Rev. Condens. Matter Phys. 7, 195 (2016).

10. Singh, Y. et al. Relevance of the Heisenberg-Kitaev model for the honeycomb lattice iridates $\mathrm{A}_{2} \mid \mathrm{rO}_{3}$. Phys. Rev. Lett. 108, 127203 (2012).

11. Plumb, K. et al. $a-\mathrm{RuCl}_{3}$ : a spin-orbit assisted Mott insulator on a honeycomb lattice. Phys. Rev. B 90, 041112 (2014).

12. Kim, H.-S. et al. Kitaev magnetism in honeycomb $\mathrm{RuCl}_{3}$ with intermediate spinorbit coupling. Phys. Rev. B 91, 241110 (2015).

13. Modic, K. A. et al. Realization of a three-dimensional spinanisotropic harmonic honeycomb iridate. Nat. Commun. 5, 4203 (2014).

14. Takayama, T. et al. Hyperhoneycomb iridate $\beta-\mathrm{Li}_{2} \mid \mathrm{rO}_{3}$ as a platform for Kitaev magnetism. Phys. Rev. Lett. 114, 077202 (2015).

15. Rau, J. G., Lee, E. K.-H. \& Kee, H.-Y. Generic spin model for the honeycomb iridates beyond the Kitaev limit. Phys. Rev. Lett. 112, 077204 (2014).

16. Ran, K. et al. Spin-wave excitations evidencing the Kitaev interaction in single crystalline $a-\mathrm{RuCl}_{3}$. Phys. Rev. Lett. 118, 107203 (2017).

17. Wang, W., Dong, Z.-Y., Yu, S.-L. \& Li, J.-X. Theoretical investigation of magnetic dynamics in a-RuCl . Phys. Rev. B 96, 115103 (2017).

18. Kim, H.-S. \& Kee, H.-Y. Crystal structure and magnetism in $a-\mathrm{RuCl}_{3}$ : an ab initio study. Phys. Rev. B 93, 155143 (2016).

19. Winter, S. M., Li, Y., Jeschke, H. O. \& Valenti, R. Challenges in design of Kitaev materials: magnetic interactions from competing energy scales. Phys. Rev. $B$ 93, 214431 (2016).

20. Yadav, R. et al. Kitaev exchange and field-induced quantum spin-liquid states in honeycomb a-RuCl . Sci. Rep. 6, 37925 (2016).

21. Rau, J. G. and Kee, H. -Y. Trigonal distortion in the honeycomb iridates: proximity of zigzag and spiral phases in $\mathrm{Na}_{2} \mathrm{IrO}_{3}$. Preprint arXiv:1408.4811 (2014).

22. Rousochatzakis, I. \& Perkins, N. B. Classical spin liquid instability driven by offdiagonal exchange in strong spin-orbit magnets. Phys. Rev. Lett. 118, 147204 (2018).

23. Imada, M. \& Takahashi, M. Quantum transfer Monte Carlo method for finite temperature properties and quantum molecular dynamics method for dynamical correlation functions.J. Phys. Soc. Jpn. 55, 3354 (1986).

24. Jaklic, J. \& Prelovsek, P. Lanczos method for the calculation ofinite-temperature quantities in correlated systems. Phys. Rev. B 49, 5065 (1994).

25. Hams, A. \& De Raedt, H. Fast algorithm for finding the eigenvalue distribution of very large matrices. Phys. Rev. E 62, 4365 (2000).

26. Sugiura, S. \& Shimizu, A. Thermal pure quantum states at finite temperatures Phys. Rev. Lett. 108, 240401 (2012).

27. Sugiura, S. \& Shimizu, A. Canonical thermal pure quantum state. Phys. Rev. Lett 111, 010401 (2013)

28. Chaloupka, J. \& Khaliullin, G. Hidden symmetries of the extended KitaevHeisenberg model: implications for the honeycomb-lattice iridates A2IrO3. Phys. Rev. B 92, 024413 (2015).

29. Sears, J. A. et al. Magnetic order in $a-\mathrm{RuCl}_{3}$ : a honeycomb-lattice quantum magnet with strong spin-orbit coupling. Phys. Rev. B 91, 144420 (2015).

30. Johnson, R. D. et al. Monoclinic crystal structure of $a-\mathrm{RuCl}_{3}$ and the zigzag antiferromagnetic ground state. Phys. Rev. B 92, 235119 (2015).

31. Cao, $\mathrm{H}$. et al. Low-temperature crystal and magnetic structure of $a-\mathrm{RuCl}_{3}$. Phys Rev. B 93, 134423 (2016)

32. Nasu, J., Udagama, M. \& Motome, Y. Thermal fractionalization of quantum spins in a Kitaev model: temperature-linear specific heat and coherent transport of Majorana fermions. Phys. Rev. B 92, 115122 (2015).

33. Yamaji, Y. et al. Clues and criteria for designing a Kitaev spin liquid revealed by thermal and spin excitations of the honeycomb iridate $\mathrm{Na}_{2} \mathrm{IrO}_{3}$. Phys. Rev. $\mathrm{B} \mathbf{9 3}$ 174425 (2016)

34. Kitaev, A. Anyons in an exactly solved model and beyond. Ann. Phys. 321, 2 (2006).

35. Knolle, J., Kovrizhin, D., Chalker, J. \& Moessner, R. Dynamics of a two-dimensional quantum spin liquid: signatures of emergent majorana fermions and fluxes. Phys. Rev. Lett. 112, 207203 (2014). 
36. Vidal, G. Classical simulation of infinite-size quantum lattice systems in one spatial dimension. Phys. Rev. Lett. 98, 070201 (2007).

37. Kimchi, l., Analytis, J. G. \& Vishwanath, A. Three-dimensional quantum spin liquids in models of harmonic-honeycomb iridates and phase diagram in an infinite-D approximation. Phys. Rev. B 90, 205126 (2014).

38. Banerjee, A. et al. Neutron scattering in the proximate quantum spin liquid $a-$ $\mathrm{RuCl}_{3}$. Science 356, 1055-1059 (2017).

39. Gohlke, M., Wachtel, G., Yamaji, Y., Pollmann, F. \& Kim, Y. B. Quantum spin liquid signatures in Kitaev-like frustrated magnets. Phys. Rev. B 97, 075126 (2018).

40. Chaloupka, J., Jackeli, G. \& Khaliullin, G. Kitaev-Heisenberg model on a honeycomb lattice: possible exotic phases in iridium oxides A2IrO3. Phys. Rev. Lett. 105, 027204 (2010).

41. Kawamura, M. et al. Quantum lattice model solver HФ. Comput. Phys. Commun. 217, 180 (2017).
Open Access This article is licensed under a Creative Commons Attribution 4.0 International License, which permits use, sharing, adaptation, distribution and reproduction in any medium or format, as long as you give appropriate credit to the original author(s) and the source, provide a link to the Creative Commons license, and indicate if changes were made. The images or other third party material in this article are included in the article's Creative Commons license, unless indicated otherwise in a credit line to the material. If material is not included in the article's Creative Commons license and your intended use is not permitted by statutory regulation or exceeds the permitted use, you will need to obtain permission directly from the copyright holder. To view a copy of this license, visit http://creativecommons. org/licenses/by/4.0/.

(c) The Author(s) 2018 